\title{
Reliability Analysis of Circuit Performance Based on Random Set Theroy
}

\author{
Xiaobin Xu ${ }^{1}$ Chenglin Wen ${ }^{2}$ Rongli Liu ${ }^{2}$ \\ ${ }^{1}$ Department of Electrical and Automation, Shanghai Maritime University, Shanghai 200135, P.R.China \\ ${ }^{2}$ School of Automation, Hangzhou Dianzi University, Hangzhou 310018, P.R.China
}

\begin{abstract}
Circuit performance is a function of circuit parameters. If these parameters are random variables, the performance index is also a random variable. System performance can be determined by value of this index and more importantly by the probability that it is maintained in specified tolerance range. To evaluate reliability of circuit performance is to analyze tolerance range and corresponding probability in order to determine robustness of circuit design. In this paper, random set theory is used to describe these parameter variables and provide a simple and flexible method for evaluating reliability of circuit performance, which is alternative to Monte-Carlo analysis, but reduces the number of calculations drastically.
\end{abstract}

Keywords: Random set theory, Reliability of circuit performance, Monte-Carlo analysis, Tolerance analysis.

\section{Introduction}

The component parameters fluctuations trigger variations in circuit performance. Such fluctuations may not result in faulty behavior, but, the circuit does not execute some given functions any longer. From the view of reliability, the system has been in failure state.Therefore, reliability of circuit performance can be determined not only by value of this performance index, but also, what's more, by the probability that it is maintained in the specified tolerance range of performance variations. Even if system has a high value of index, the low probability indicates that system no more retains satisfactory functional behavior. So it is necessary to estimate tolerance range and the corresponding probability in order to determine the robustness of the circuit design [1]-[2].

The circuit performance is a function of circuit component parameters. Typical parameters are component values, such as resistances, capacitances, inductances,etc. Statistical variations in each component value result in parameter variation, possibly centered around the nominal value (design center) for each component. Namely, if parameters are random variables, the performance index is also a random variable. The performance function may vary from simple linear form to complex nonlinear form.

We want to know the distribution of performance index for evaluating reliability of circuit performance. Monte Carlo analysis provides a means for doing this[3]-[4]. However, when the variance of variable estimated is quite large or the small estimation error is given, the large number of simulations required prevents its application[1]. It is well known that substantial computational savings can be obtained by using advance Monte Carlo methods such as "Importance sampling", "Stratified sampling", "Correlated sampling” techniques etc. Whereas, very great skills are required in applications. For examples, one must obtain optimal sampling functions partly by guesswork in importance sampling. Such guessing is not a easy job. How to stratify is a bottleneck in stratified sampling, so they can not be used widely.

Random set theory provides a general mechanism for describing and handing many kinds of information (random, vague, imprecise, etc)[5]-[6]. In this paper, random set theory is used to describe these parameter variables and present a simple and flexible method for evaluating reliability of circuit performance, which is alternative to Monte-Carlo analysis, but the computational complexity relates not to variance of variable, but to choice of random sets. So the proposed method can reduces the number of calculations required drastically.

This paper is organized as follow: section 2 recalls some notions about random sets, along with the principles that allow random-set-type information on parameters to be extended to the circuit performance index. A model for analyzing reliability of circuit performance is constructed in section 3. Based on this model, section 4 details the random set method for reliability analysis. An application of proposed method is given in sections 5 to prove it effective. By compareing it with Monte Carlo method, drastic computational savings are showed. 


\section{Random set and the correspond- ing principles}

\subsection{Random set and random rela- tion}

Let $U$ be a non-empty set.

Definition 1[7] A finite support random set on $U$ is a pair $(\mathcal{F}, m)$ where $\mathcal{F}$ is a finite family of distinct non-empty subsets of $U$ and $m$ is a mapping $\mathcal{F} \rightarrow[0,1]$ and such that $\sum_{A \in \mathcal{F}} m(A)=1 . \mathcal{F}$ is called the support of the random set and $m$ is called a basic probability assignment. A subset $A$ with a non-null mass is viewed as a focal element.

Let a parameter $\xi$ be a random variable, if each set $A \in \mathcal{F}$ contains the possible values of $\xi, m(A)$ can be viewed as the probability that $A$ is the actual range of $\xi$. Such a random set $(\mathcal{F}, m)$ is equivalent to a belief function in the sense of Shafer [8] Given a random set $(\mathcal{F}, m)$, a belief function (Bel) can be defined as the following set function

$$
\forall A \subseteq U \quad \operatorname{Bel}(A)=\sum_{B \subseteq A} m(B)
$$

A corresponding plausibility function $(\mathbf{P I})$ is

$$
\operatorname{Pl}(A)=1-\operatorname{Bel}\left(A^{c}\right)=\sum_{A \cap B \neq \varnothing} m(B)
$$

Where $A^{c}$ denotes the complement of $A$. Contrarily, using Moebius inversion, we can reconstruct $(\mathcal{F}, m)$ from the knowledge of the set function Bel.

$$
m(A)=\sum_{B \subseteq A}(-1)^{|A-B|} \operatorname{Bel}(B)
$$

Definition 2[9] A multi-dimensional space $U=$ $U_{1} \times \cdots \times U_{n}$, where $\times$ indicates Cartesian product. A finite support random relation is a random set $(\mathcal{F}, m)$ on $U$.

The projections of a random relation on Cartesian product $U_{1} \times \cdots \times U_{n}$ are defined by Shafer to be the marginal random set $\left(\mathcal{F}_{k}, m_{k}\right), k=1, \cdots, n$ [8]

$$
\forall C_{k} \subseteq U_{k}, m_{k}\left(C_{k}\right) \square \sum\left\{m(A) \mid C_{k}=\operatorname{Proj}_{U_{k}}(A)\right\}
$$

$\operatorname{Proj}_{U_{k}}(A)=\left\{u_{k} \in U_{k} \mid \exists u=\left(u_{1}, \cdots, u_{k}, \cdots u_{n}\right) \in A\right\}$ (2.5)

\subsection{Extension principles}

Let $\xi=\left(\xi_{1}, \cdots, \xi_{n}\right)$ be a variable on $U=$ $U_{1} \times \ldots \times U n, \zeta=f(\xi), f: U \rightarrow V$ is function of $\xi$. The random set $(\mathcal{R}, \rho)$ of $\zeta$, which is the image of random relation $(\mathcal{F}, m)$ of $\xi$ through $f$, is given by extension principles [7]

$$
\begin{gathered}
\mathcal{R}=\left\{R_{j}=f\left(A_{i}\right) \mid A_{i} \in \mathcal{F}\right\} \\
\rho\left(R_{j}\right)=\sum\left\{m\left(A_{i}\right) \mid R_{j}=f\left(A_{i}\right)\right\}
\end{gathered}
$$

where

$$
f\left(A_{i}\right)=\left\{f(u) \mid u \in A_{i}\right\}, i=1, \cdots, M
$$

$M$ is the number of element of $\mathcal{F}$. The summation in Eq.(2.7) account for the fact that more than one focal element $A_{i}$ may yield the same image $R_{j}$ through $f$.

Specially, if $\xi$ is a discrete random variable and every focal element $A_{i}$ contains only one element, $m$ defines a standard joint probability mass function on $U$ and Eq.(2.7) gives $\rho$ on $V$, which also is the probability mass function $p_{\zeta}(y)$ according to usual probability theory

$$
\rho(y)=p_{\zeta}(y)=\sum\{m(u) \mid y=f(u)\}
$$

The basic step to construct $(\mathcal{R}, \rho)$ is to calculate the image of focal elements through $f$. In general, this problem can be solved by applying twice the technique of global optimization [10]. According to properties of $f$, we discuss the calculation of the image of a random relation as follow

If $A \in \mathcal{F}$ is a finite closed set and $f$ is a continuous function, then

$$
f(A)=[a, b]
$$

where $a=\min _{u \in A}(f(u)), b=\max _{u \in A}(f(u))$.

If each component $\xi_{k}(k=1, \cdots, n)$ of $\xi$ corresponds to a marginal random set $\left(\mathcal{F}_{k}, m_{k}\right)$, whose focal element is a interval $\left[l_{k}, r_{k}\right]$, the focal element of $(\mathcal{F}, m)$ can be obtained by

$$
A=C_{1} \times \cdots \times C_{n}=\left[l_{1}, r_{1}\right] \times \cdots \times\left[l_{n}, r_{n}\right] \quad \text { (2.11) }
$$
In this case, the methods of interval analysis are applicable [11]. If $A$ is a convex set, it has $2^{n}$ vertices, denoted as $v_{j}, j=1, \cdots, 2^{n}$.when $f$ possesses some properties, the vertex method can help to reduce the computational burden considerably [12]. The vertex method may be showed as follows

Proposition 1 if $\zeta=f(\xi)$ is continuous in $A$, and also no extreme point exists in this region (including its boundaries), then the value of interval function can be obtained by

$$
\begin{aligned}
f(A)= & {\left[\min _{j}\left\{f\left(v_{j}\right): j=1, \cdots, 2^{n}\right\},\right.} \\
& \left.\max _{j}\left\{f\left(v_{j}\right): j=1, \cdots, 2^{n}\right\}\right]
\end{aligned}
$$

So, $f$ has to be calculated $2^{n}$ times for each focal element $A_{i}$. This computational burden can be further reduced if the hypotheses of the following proposition hold [13]

Proposition 2 if $f$ and its partial derivatives are all continuous and if $f$ is a strictly monotonic function with regard to each parameter variable $\xi_{k}(k=1, \cdots, n)$, then

$$
\begin{aligned}
& \exists v_{\text {min }}: f\left(v_{\text {min }}\right)=\min _{j}\left\{f\left(v_{j}\right): j=1, \cdots, 2^{n}\right\} \\
& \exists v_{\text {max }}: f\left(v_{\text {max }}\right)=\max _{j}\left\{f\left(v_{j}\right): t=1, \cdots, 2^{n}\right\}
\end{aligned}
$$




\subsection{Inclusions of random sets and monotonicity principle}

Yager defined the inclusion of random set [14]

Definition $3\left(\mathcal{F}_{1}, m_{1}\right) \subseteq\left(\mathcal{F}_{2}, m_{2}\right)$ if and only if the three following conditions hold

(1) For $\forall A \in \mathcal{F}_{1}, \exists B \in \mathcal{F}_{2}$, such that $A \subseteq B$;

(2) For $\forall B \in \mathcal{F}_{2}, \exists A \in \mathcal{F}_{1}$, such that $A \subseteq B$;

(3) There is a non-negative assignment matrix $W$ with entries $W(A, B)$ satisfying

$$
\begin{aligned}
& \forall A \in \mathcal{F}_{1}, m_{1}(A)=\sum_{B: A \subseteq B} W(A, B) \\
& \forall B \in \mathcal{F}_{2}, m_{2}(B)=\sum_{A: A \subseteq B} W(A, B)
\end{aligned}
$$

where, if $A \not \subset B, W(A, B)=0$. In other word, the weights $m_{1}(A)$ can be shared among supersets of $A$ which belong to $\mathcal{F}_{2}$, and the weight $m_{2}(B)$ is the sum of the shares allocated to subsets of $B$.

According to this inclusion between two random sets, the relationship between their Bel and Pl function can be induced by [14]

$$
\begin{aligned}
& \left(\mathcal{F}_{1}, m_{1}\right) \subseteq\left(\mathcal{F}_{2}, m_{2}\right) \Rightarrow \\
& {\left[\operatorname{Bel}_{1}(A), \mathrm{Pl}_{1}(A)\right] \subseteq\left[\operatorname{Bel}_{2}(A), \mathrm{Pl}_{2}(A)\right]}
\end{aligned}
$$

Based on extension principle in section 2.2, this inclusion property can be transformed to the image of random relations, which is monotonicity principle [7]

Proposition 3 let $(\mathcal{F}, m),\left(\mathcal{F}^{\prime}, m^{\prime}\right)$ be two random relations on and $f$ be a function $U \rightarrow V$. Let $(\mathcal{R}, \rho)$, $\left(\mathcal{R}^{\prime}, \rho^{\prime}\right)$ be the respective images of $(\mathcal{F}, m),\left(\mathcal{F}^{\prime}, m^{\prime}\right)$ through $f$. Then $(\mathcal{R}, \rho) \subseteq\left(\mathcal{R}^{\prime}, \rho^{\prime}\right)$ as soon as $(\mathcal{F}, m) \subseteq(\mathcal{F}, m)$.

Let $\operatorname{Bel}(\cdot), \operatorname{Pl}(\cdot)$ and $\operatorname{Bel}^{\prime}(\cdot), \operatorname{Pl}^{\prime}(\cdot)$ are the belief and plausibility function of $(\mathcal{R}, \rho),\left(\mathcal{R}^{\prime}, \rho^{\prime}\right)$ respectively. Then, by means of Eq.(2.17)

$$
[\operatorname{Bel}(\cdot), \mathrm{Pl}(\cdot)] \subseteq\left[\operatorname{Bel}^{\prime}(\cdot), \mathrm{Pl}^{\prime}(\cdot)\right]
$$

Dobios and Prade firstly indicated how inclusions of random set might be used to bound the cumulative distribution function (CDF) of a sum of two random variables [7].This means that, if the actual information about event $A$ can be known under the form of a random set $(\mathcal{R}, \rho)$, it is possible to find other $\left(\mathcal{R}^{\prime}, \rho^{\prime}\right)$ which can include $(\mathcal{R}, \rho)$. When the computation of $\left(\mathcal{R}^{\prime}, \rho^{\prime}\right)$ is much simper than $(\mathcal{R}, \rho)$, the obtained intervals $\left[\operatorname{Bel}^{\prime}(A), \mathrm{Pl}^{\prime}(A)\right]$ are a bracketing of $[\operatorname{Bel}(A), \operatorname{Pl}(A)]$ which may be enough for making a decision about the event $A$ of interest.

In reliability analysis of circuit performance, performance functions are the circuit outputs $\zeta=f(\xi)$. $\xi=\left(\xi_{1}, \cdots ; \xi_{n}\right), \xi_{i}$ is parameter of $i$ th unit or component. If $\xi_{i}$ is random variable, $\zeta$ is also random variable. To evaluate reliability of circuit performance is to analyze whether the probability that $\zeta$ fluctuates in the specific tolerance range is acceptable. In this paper, by virtue of monotonicity principle and vertex methods, the idea of bounding CDF of a variable in reference [7] is generalized to provide the upper and lower bounds of the probability distribution of performance index $\zeta$. Where $f$ can vary from simple linear form to complex nonlinear form and $\xi$ is generic random vector with various distribu-tions. Using this upper and lower bound, reliability of circuit performance can be determined easily. In following section, we construct the analysis model, based on which, the random set method for analyzing reliability is presented in detail.

\section{Reliability analysis model of cir- cuit performance}

Suppose that the circuit system is composed of $n$ units $S_{1}, \cdots, S_{n}, u_{1}, \cdots, u_{n}$ are respective parameters of units, the system performance is

$$
y=f(u)
$$

$u=\left(u_{1}, \cdots, u_{n}\right)$ is the parameter vector.

As a rule, the nominal value of $k$ th parameter is denoted as $u_{k_{-} 0}$, its tolerance is $\pm \Delta u_{k}$.In another word,

$$
u_{k_{-} 0}+\Delta u_{k} \geq u_{k} \geq u_{k_{-} 0}-\Delta u_{k}
$$

Generally, let the parameter of $S_{k}$ be a random variable $\xi_{k}$. When it is normal distribution variable, its probability density function is given by

$$
p_{\xi_{k}}\left(u_{k}\right)=\frac{1}{\sqrt{2 \pi \sigma}} \exp \left[-\frac{\left(u_{k}-\mu_{k}\right)}{2 \sigma_{k}^{2}}\right]
$$

where $\mu_{k}=u_{k_{-} 0}, \sigma_{k}=\Delta u_{k} / 3$.

Indeed, in some cases, parameter $\xi_{k}$ is not limited to normal distribution and may be other types of distribution. If $u_{k}$ is viewed as random variable $\xi_{k}$, performance index $y$ is also a random variable denoted as $\zeta$

Commonly, the tolerance range of system performance index is given in advance, as a interval $D=\left[y_{A}, y_{B}\right]$, thus, the cumulative probability of $\zeta \in D$ is

$$
\begin{aligned}
P_{\zeta}(D) & =P_{\zeta}\left(y_{A} \leq \zeta \leq y_{B}\right) \\
= & F_{\zeta}\left(y_{B}\right)-F_{\zeta}\left(y_{A}\right)=\int_{y_{A}}^{y_{B}} p_{\zeta}(y) d y
\end{aligned}
$$

If the probability of failure of circuit performance is given as $\Upsilon$, such that evaluation criterion can be defined as follow

(1)If $P_{\zeta}\left(y_{A} \leq \zeta \leq y_{B}\right)>\Upsilon$, system performance is reliable.

(2)If $P_{\zeta}\left(y_{A} \leq \zeta \leq y_{B}\right)<\Upsilon$, system performance is unreliable.

(3)If $P_{\zeta}\left(y_{A} \leq \zeta \leq y_{B}\right)=\Upsilon$, system performance is in a limit state condition.

\section{The random set method for relia- bility analysis}


If the performance index $\zeta=f(\xi)$ is known under the form of a random relation $(\mathcal{F}, m)$, where every focal element $A_{i}$ contains only one element $A=\{u\}$, $m(\{u\})$ is equivalent to probability mass function $p_{\zeta}(u)$. Based on such $(\mathcal{F}, m)$, we can obtain $\left(\mathcal{F}^{\prime}, m^{\prime}\right)$ by inclusion of random set, using extension principle, $\left(\mathcal{R}^{\prime}, \rho^{\prime}\right)$ can be obtained. And then, according to monotonicity principle, we can get $(\mathcal{R}, \rho) \subseteq\left(\mathcal{R}^{\prime}, \rho^{\prime}\right)$. Finally, $\operatorname{Bel}^{\prime}(\cdot), \operatorname{Pl}^{\prime}(\cdot)$ is used to construct upper and lower bounds of $\mathrm{CDF}_{\zeta}(y)$ yielded by Monte Carlo simulation, on the basis of model introduced in section 3, reliability of circuit performance is determined by the upper and lower bounds. The whole procedure is detailed in follow section.

\subsection{The procedure of the random set method}

1) The calculation of $(\mathcal{F}, m)$ and $\left(\mathcal{F}^{\prime}, m^{\prime}\right)$

From Eq.(3.2), we know that physical parameter $\xi_{k}$ belongs to interval $I_{k}=\left[u_{k_{-} 0}+\Delta u_{k}, u_{k_{-} 0}-\Delta u_{k}\right]$. Let $\bar{U}=I_{1} \times \cdots \times I_{n}$, thus, $\xi=\left(\xi_{1}, \cdots, \xi_{n}\right) \in \bar{U}$. Let $\mathcal{F}=\bar{U}$, $m=p_{\xi}$ (every focal element of single-value). We set that $\mathcal{F}=\{A, i=1, \cdots, M\}$ which is a partition of $\bar{U}$, accordingly, $I_{k}$ is partition into $d_{k}$ subintervals, thus $A_{i}=C_{1}^{i} \times C_{2}^{i} \times \cdots \times C_{n}^{i}, C_{k}^{i} \subseteq I_{k} \quad k=1, \cdots, n, i=1, \cdots, M(4.1)$ Where $M$ is the number of elements of $\mathcal{F}^{\prime}$

$$
M=\prod_{k=1}^{n} d_{k}
$$

Let

$$
\begin{gathered}
m^{\prime}\left(A_{i}\right)=\sum_{u: u \in A_{i}} p_{\xi}(u) \\
W\left(u, A_{i}\right)=p_{\xi}(u), \quad \text { if } u \in A_{i} \\
W\left(u, A_{i}\right)=0, \quad \text { if } u \notin A_{i}
\end{gathered}
$$

We can prove that $(\mathcal{F}, m)$ and $\left(\mathcal{F}^{\prime}, m^{\prime}\right)$ satisfy three conditions in definition 3.

(1) For $\forall u \in \mathcal{F}$, necessarily, $\exists A \in \mathcal{F}$, such that $u \subseteq A_{i}$.

(2)For $\forall A_{i} \in \mathcal{F}^{\prime}$, at least one $u$ exists such that $u \subseteq A_{\text {. }}$.

(3) $m^{\prime}\left(A_{i}\right)=\sum_{u: u \in A_{i}} p_{\xi}(u)=\sum_{u: u \in A_{i}} W\left(u, A_{i}\right)$

$m(u)=p_{\xi}(u)=\sum_{A_{i}: u \in A_{i}} W\left(u, A_{i}\right)$

So, $(\mathcal{F}, m) \subseteq\left(\mathcal{F}^{\prime}, m^{\prime}\right)$. If $\xi$ is a continuous random variable, $p_{\xi}(u)$ is joint probability density function (PDF) and Eq.(4.3) is changed into

$$
m^{\prime}\left(A_{i}\right)=\int_{A_{i}} p_{\xi}(u) d u
$$

2) The calculation of the image $\left(\mathcal{R}^{\prime}, \rho^{\prime}\right)$ of $\left(\mathcal{F}^{\prime}, m^{\prime}\right)$ After constructing $\left(\mathcal{F}^{\prime}, m^{\prime}\right)$, we can get its image $\left(\mathcal{R}^{\prime}, \rho^{\prime}\right)$ by means of extension principle

$$
\begin{gathered}
\mathcal{R}^{\prime}=\left\{R_{j}=f\left(A_{i}\right) \mid A_{i} \in \mathcal{F}^{\prime}\right\} \\
f\left(A_{i}\right)=\left\{f(u) \mid u \in A_{i}\right\}
\end{gathered}
$$

Actually, $A_{i}$ is a $n$-dimensional boxes, whose number of vertices is $v$

$$
v=\prod_{k=1}^{n}\left(d_{k}+1\right)
$$

If $f$ satisfies the conditions of proposition 1 and 2, the calculation of $f\left(A_{i}\right)$ will become feasible. For example, $n=2, d_{1}=3, d_{2}=2$, thus, $V=I_{1} \times I_{2} . I_{1}$ is partitioned into three subintervals $\left[u_{1,1}, u_{1,2}\right),\left[u_{1,2}, u_{1,3}\right)$, $\left[u_{1,3}, u_{1,4}\right], I_{2}$ is partitioned into two subintervals $\left[u_{2,1}, u_{2,2}\right),\left[u_{2,2}, u_{2,3}\right]$. The entire focal elements are given: $A=\left[u_{1,1}, u_{1,2}\right) \times\left[u_{2,1}, u_{2,2}\right), A_{2}=\left[u_{1,1}, u_{1,2}\right) \times\left[u_{2,2}, u_{2,3}\right]$, $A_{3}=\left[u_{1,2}, u_{1,3}\right) \times\left[u_{2,1}, u_{2,2}\right), A_{4}=\left[u_{1,2}, u_{1,3}\right) \times\left[u_{2,2}, u_{2,3}\right)$, $A_{5}=\left[u_{1,3}, u_{1,4}\right] \times\left[u_{2,1}, u_{2,2}\right), A_{6}=\left[u_{1,3}, u_{1,4}\right] \times\left[u_{2,2}, u_{2,3}\right]$ so, $M=6, v=12$.

If $f$ and its partial derivatives are all continuous on $I_{1}$ and $I_{2}, f$ is increasing on $I_{1}$ and is decreasing on $I_{2}$, according to proposition 2, for example, the image of $A_{2}$ is

$f\left(A_{2}\right)=\left[\min \left\{f\left(u_{1,1}, u_{2,3}\right)\right\}, \max \left\{f\left(u_{1,2}, u_{2,2}\right)\right\}\right]$

Because the vertex $\left(u_{1,1}, u_{2,1}\right)$ of $A_{1}$ and the vertex $\left(u_{1,4}, u_{2,3}\right)$ of $A_{6}$ are not the extreme points of $f$, and there are no this two points in the contiguous focal elements with $A_{1}$ and $A_{6}$, then the times of calculating all image is reduced to $(3+1) \times(2+1)-2=10$.

Form Eq. (2.7), we can get

$$
\rho^{\prime}\left(R_{j}\right)=\sum\left\{m^{\prime}\left(A_{i}\right) \mid R_{j}=f\left(A_{i}\right)\right\}
$$

\section{3) The construction of upper and lower bound of $F_{\zeta}$}

For $\forall R \in \mathcal{R}^{\prime}$ Bel and Pl of $\left(\mathcal{R}^{\prime}, \rho^{\prime}\right)$ can be obtained by Eq.(2.1) and Eq.(2.2)

$$
\begin{gathered}
\operatorname{Bel}^{\prime}(R)=\sum_{Q \subseteq R} \rho^{\prime}(Q)=\sum_{Q \subseteq R} \sum_{Q=f\left(A_{i}\right)} m^{\prime}\left(A_{i}\right) \\
\operatorname{Pl}^{\prime}(R)=\sum_{Q \cap R \neq \varnothing} \rho^{\prime}(Q)=\sum_{R \cap Q \neq \varnothing} \sum_{Q=f\left(A_{i}\right)} m^{\prime}\left(A_{i}\right)
\end{gathered}
$$

In evidence theory [8], $\operatorname{Bel}^{\prime}(R)$ and $\operatorname{Pl}^{\prime}(R)$ are respective defined as upper and lower bound of probability $P_{\zeta}(R)$, such that

$$
\operatorname{Bel}^{\prime}(R) \leq P_{\zeta}(R) \leq \mathrm{Pl}^{\prime}(R)
$$

Further, the upper and lower CDFs of $\zeta$ are respective $F_{\zeta}^{*}(y)=\operatorname{Pl}((-\infty, y])=\sum\left\{\rho\left(f\left(A_{)}\right)\right) \mid y \geq \inf \left(f\left(A_{f}\right)\right)\right\}(4.14)$ $F_{*}^{\zeta}(y)=\operatorname{Bel}((-\infty, y])=\sum\left\{\rho\left(f\left(A_{i}\right)\right) \mid y \geq \sup \left(f\left(A_{i}\right)\right)\right\}$ 
Obviously,

$$
F_{*}^{\zeta}(y) \leq F_{\zeta}(y) \leq F_{\zeta}^{*}(y)
$$

That is to say, both of them can bracket the actual distribution of variable. Usually, in practice, the CDF obtained by Monte Carlo simulation is used as approximation of the actual one.

4) The reliability analysis by virtue of the upper and lower CDFs

On the basis of reliability analysis model in section 3, if the tolerance range is $D=\left[y_{A}, y_{B}\right]$, the proba-bility of $\zeta \in D$ is

$$
P_{\zeta}(D)=F_{\zeta}\left(y_{B}\right)-F_{\zeta}\left(y_{A}\right)
$$

Let

$$
\begin{aligned}
& P_{*}^{\zeta}(D)=F_{*}^{\zeta}\left(y_{B}\right)-F_{\zeta}^{*}\left(y_{A}\right) \\
& P_{\zeta}^{*}(D)=F_{\zeta}^{*}\left(y_{B}\right)-F_{*}^{\zeta}\left(y_{A}\right)
\end{aligned}
$$

We can conclude

$$
P_{*}^{\zeta}(D) \leq P_{\zeta}(D) \leq P_{\zeta}^{*}(D)
$$

The evaluation criterion can be defined as follow

(1) If $P_{*}^{\zeta}(D)>\Upsilon$, from Eq.(4.21), $P_{\zeta}(D)>\Upsilon$,system performance is reliable.

(2) If $P_{*}^{\zeta}(D)<\Upsilon, P_{\zeta}(D)<\Upsilon$, system performance is unreliable.

When $P_{*}^{\zeta}(D)<\Upsilon<P_{\zeta}^{*}(D)$, the reason for it maybe is an excessive coarse partition to $V$. There are unacceptable large gaps between $F_{\zeta}(y)$ and $F_{*}^{\zeta}(y), F_{\zeta}^{*}(y)$. In this case, we may set the error of calculating $F_{\zeta}(y)$ and $F_{*}^{\zeta}(y)$ in advance, and then divide $V$ into more subintervals, obtain new $F_{\zeta}(y)$ and $F_{*}^{\zeta}(y)$. If $P_{*}^{\zeta}(D)<\Upsilon<P_{\zeta}^{*}(D)$ again, we can conclude that system performance is in a limit state condition.

\subsection{Error comparison between the random set method and Monte Carlo method}

Besides much less computational burden than Monte Carlo simulation, the precision of the proposed method can be explicitly evaluated by given forms of error.

$$
\begin{aligned}
& \text { Maximum Error } \\
& \qquad \varepsilon_{-} \operatorname{ran}_{\max }=\max _{y}\left(F_{\zeta}^{*}(y)-F_{*}^{\zeta}(y)\right)
\end{aligned}
$$

$$
\begin{aligned}
& \text { Local Error } \\
& \qquad \varepsilon_{-} \operatorname{ran}(y)=F_{\zeta}^{*}(y)-F_{*}^{\zeta}(y) \quad \forall y \in Y
\end{aligned}
$$

In practice, according to different cases, both of them can be chose to evaluate gaps between the actual distribution and upper and lower CDF from global and local aspects respectively.
In Monte Carlo methods, the likely deviation from $\mathrm{CDF}_{\zeta}(y)$ obtained by Monte Carlo simulation to the actual distribution $F_{T r_{-} \zeta}(y)$ is an envelop around the latter with a certain confidence level, but not a guarantee [15]. However, the present method can provide this guarantee, i.e.100\% confidence level. When the confidence level $1-\alpha$,confidence level, $\lambda_{\alpha}$ and variance $\sigma^{2}$ of variable are respectively given, the error of Monte Carlo simulation can be defined as $\varepsilon_{-}$mon [1]

$$
\frac{\lambda_{\alpha} \sigma}{\sqrt{N}} \leq \varepsilon_{-} \text {mon }
$$

So, the number of sampling must satisfies

$$
N \geq\left(\frac{\lambda_{\alpha} \sigma}{\varepsilon_{-} m o n}\right)^{2}
$$

Obviously, when the variance of variable estimated is quite large or the small estimation error is given, the large number of simulations required prevents its application [1]. It is well known that substantial compu-tational savings can be obtained by using advance Monte Carlo methods such as "Importance Sampling”, "Stratified Sampling”, "Correlated Sampling” tech-niques etc, but, very great skills are required in appli-cations. So they can not be used widely.

For the proposed procedure, the computation load is only determined by partition of tolerance range of each circuit parameter. When $f$ has some properties, the vertex methods can help to reduce the computation burden sharply, and the partitions of tolerance range are flexible and convenient. In follow section, a example is given to prove it effective.

\section{Data analysis and application results}

To evaluate a familiar RLC Series Resonance Circuit in Fig 1, it is required that the nominal value of the resonance frequency output $f_{0}$ is $4110 \mathrm{kHz}$, the maximum frequency drift is limited to $\pm 100 \mathrm{k} \mathrm{Hz}$. The reliability of performance must be greater than or equal to $\Upsilon=0.95$. The chose inductor $L=50 \pm 10 \% \mu \mathrm{H}$ and capacitor $C=30 \pm 5 \% \mathrm{PF}$. Suppose that the deviations of $L$ and $C$ are all normal distribution, mutually independent and the standard variances are given respectively as $\sigma_{L}=5 / 3 \mu \mathrm{H}, \sigma_{C}=0.5 \mathrm{PF}$.

Here, we analyze the reliability of this circuit performance by random set method as follow 1) Based on the model in section 3 , the performance function is the actual resonance frequency $f$ 


$$
f=\frac{10^{6}}{2 \pi\left(\xi_{1} \xi_{2}\right)^{1 / 2}}
$$

Where, $\xi_{1}=L$, mean $\mu_{\xi}=L_{0}=50 \mu \mathrm{H}$,standard variance $\sigma_{\xi_{1}}=5 / 3 \mu \mathrm{H}$; the random variable $\xi_{2}=C$, mean value $\mu_{\xi_{2}}=C_{0}=30 \mathrm{PF}$, standard variance $\sigma_{\xi_{2}}=0.5 \mathrm{PF}$.

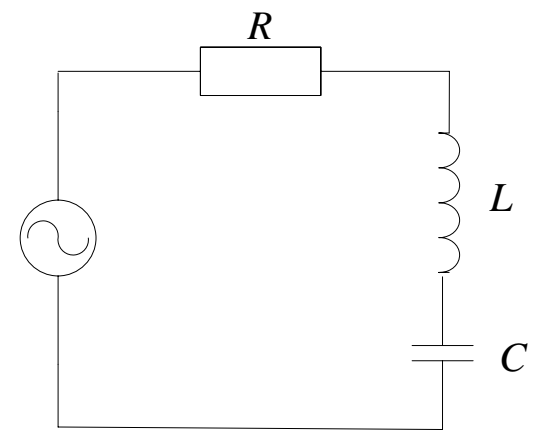

Fig 1: RLC Series Resonance Circuit

2) To describe $\xi=\left(\xi_{1}, \xi_{2}\right)$ as random relation $\left(\mathcal{F}^{\prime}, m^{\prime}\right)$, $\xi_{1}$ fluctuates in interval $I_{1}=[45,55]$, let us divide it into $d_{1}=10$ subinterval $I_{1, j}=\left[u_{1, j}, u_{1, j+1}\right),\left(j=1, \cdots, d_{1}\right), \xi_{2}$ fluctuates in interval $I_{2}=[28.5,31.5],\left(k=1, \cdots, d_{2}\right)$. $V=I_{1} \times I_{2}$ is partitioned into $M=10 \times 6=60$ focal elements, so that

$$
\mathcal{F}^{\prime}=\left\{I_{1, j} \times I_{2, k} \mid j=1, \cdots, d_{1} ; k=1, \cdots, d_{2}\right\}
$$

Because $\xi_{1}, \xi_{2}$ are mutually independent, from Eq.(4.6)

$$
\begin{aligned}
m^{\prime} & \left(I_{1, j} \times I_{2, k}\right)=\int_{I_{1, j} \times I_{2, k}} p_{\xi}\left(u_{1}, u_{2}\right) d u_{1} d u_{2} \\
& =\int_{I_{1, j} \times I_{2, k}} p_{\xi_{1}}\left(u_{1}\right) p_{\xi_{2}}\left(u_{2}\right) d S_{1} d u_{2} \\
& =\left(\int_{I_{1, j}} p_{\xi_{1}}\left(u_{1}\right) d u_{1}\right)\left(\int_{I_{2, k}} p_{\xi_{2}}\left(u_{2}\right) d u_{2}\right) \\
& =m_{1}^{\prime}\left(I_{1, j}\right) m_{2}^{\prime}\left(I_{2, k}\right)
\end{aligned}
$$

$m_{1}^{\prime}\left(I_{1, j}\right), m_{2}^{\prime}\left(I_{2, k}\right)$ are respective basic probability assignments to subintervals of $I_{1}$ and $I_{2}$, showed in table 1 and 2.

3) This step is to calculate the image $\left(\mathcal{R}, \rho^{\prime}\right)$ of $\left(\mathcal{F}, m^{\prime}\right)$. It is easy to know that $f$ and its partial derivatives are all continuous on the interval $V$. $f$ is decreasing with respect to $\xi_{1}, \xi_{2}$. According to proposition 2, to each focal element $I_{1, j} \times I_{2, k}$, the maximum of $f$ is attained when $\xi_{1}=u_{1, j}$ and $\xi_{2}=u_{2, k}$, the minimum of $f$ is attained when $\xi_{1}=u_{1, j+1}, \xi_{2}=u_{2, k+1}$. From Eq. (4.7), we have

$$
\mathcal{R}^{\prime}=\left\{R=f\left(I_{1, j} \times I_{2, k}\right) \mid j=1, \cdots, d_{1} ; k=1, \cdots, d_{2}\right\}(5.4
$$

$$
\rho^{\prime}(R)=\sum\left\{m^{\prime}\left(I_{1, j} \times I_{2, k}\right) \mid R=f\left(I_{1, j} \times I_{2, k}\right)\right\}
$$

The focal elements and the basic probability assignments of $\left(\mathcal{F}^{\prime}, m^{\prime}\right)$ and $\left(\mathcal{R}^{\prime}, \rho^{\prime}\right)$ is showed partially in table 3. By calculation, we know the images $R_{i}=f\left(I_{1, j} \times\right.$ $\left.I_{2, k}\right)$ are all distinct, so $\rho^{\prime}\left(I_{1, j} \times I_{2, k}\right)=m^{\prime}\left(I_{1, j} \times I_{2, k}\right)$.

The upper and lower cumulative distribution of $f$ are given as $F_{u p}(y)$ and $F_{\text {low }}(y)$ in Fig2, together with the CDF as $F(y)$ yielded by Monte Carlo simulation. Evidently, the latter distribution is bracketed by the upper and lower distribution.

For calculation of $F(y)$, firstly $10^{7}$ samples $\left(u_{1, l}, u_{2, l}\right) l=1, \cdots, 10^{7}$ of $\xi_{1}$ and $\xi_{2}$ is generated, whose values of function calculated are $y_{l}=f\left(\left(u_{1,1}, u_{2,1}\right)\right)$. The variance $\hat{\sigma}^{2}$ of $y_{l}$ is unbiased estimation of $\sigma^{2}$, which is considered as an approximation of the true variance $\sigma^{2}$.

We set that error $\varepsilon=0.05$,the confidence level is $95 \%$, whose $\lambda_{\alpha}=1.96$. From Eq.(4.24), $N=3536969$ samplings (simulation)is needed, however, only $(10+1) \times$ $(6+1)-2=75$ evaluations of $f$ are necessary in the random set method.

4) The last step is to evaluate the reliability of system performance by virtue of the upper and lower distribution. Based on model in section 3, the tolerance range of $f$ is $D=[4010 \mathrm{k} \mathrm{Hz}, 4210 \mathrm{k} \mathrm{Hz}]$,

The probability of failure calculated by Monte Carlo method is $P_{\zeta}(D)=0.8057$. In random set method, from

Eq.(4.14)and Eq.(4.15), we have

(1) When $\zeta=4010 \mathrm{kHz}$ :

$$
\begin{aligned}
F_{\zeta}^{*}(4010) & =\sum\left\{\rho\left(f\left(A_{i}\right)\right) \mid 4010 \geq \inf \left(f\left(A_{i}\right)\right)\right\} \\
& =0.2171 \\
F_{*}^{\zeta}(4010) & =\sum\left\{\rho\left(f\left(A_{i}\right)\right) \mid 4010 \geq \sup \left(f\left(A_{i}\right)\right)\right\} \\
& =0.0354
\end{aligned}
$$

The local error is

$$
\varepsilon_{-} r a n(4010)=F_{\zeta}^{*}(4010)-F_{*}^{\zeta}(4010)=0.1817(5.8)
$$

(2) When $\zeta=4210 \mathrm{k} \mathrm{Hz}$ :

$$
\begin{aligned}
F_{\zeta}^{*}(4210) & =\sum\left\{\rho\left(f\left(A_{i}\right)\right) \mid 4210 \geq \inf \left(f\left(A_{i}\right)\right)\right\} \\
& =0.9587 \\
F_{*}^{\zeta}(4210) & =\sum\left\{\rho\left(f\left(A_{i}\right)\right) \mid 4210 \geq \sup \left(f\left(A_{i}\right)\right)\right\} \\
= & 0.7742
\end{aligned}
$$

The local error is

$$
\varepsilon_{-} r a n(4210)=F_{\zeta}^{*}(4210)-F_{*}^{\zeta}(4210)=0.1845(5.11)
$$

The maximum error happens at $\zeta=4097.1 \mathrm{k} \mathrm{Hz}$, and $\varepsilon_{-} r a n_{\max }=0.3770$.

From Eq.(4.18)and Eq.(4.19), we have 


\begin{tabular}{c|c|c|c|c|c|c|c|c|c|c}
\hline$I_{1, j}$ & {$[45,46)$} & {$[46,47)$} & {$[47,48)$} & {$[48,49)$} & {$[49,50)$} & {$[50,51)$} & {$[51,52)$} & {$[52,53)$} & {$[53,54)$} & {$[54,55]$} \\
\hline$m_{1}{ }^{\prime}$ & 0.0068 & 0.0277 & 0.0791 & 0.1592 & 0.2257 & 0.2257 & 0.1592 & 0.0791 & 0.0277 & 0.0068 \\
\hline \multicolumn{1}{c}{ Table 1: $m_{1}{ }^{\prime}\left(I_{1, j}\right)$ BPA, $j=1, \ldots, 10$}
\end{tabular}

\begin{tabular}{c|c|c|c|c|c|c}
\hline$I_{2, k}$ & {$[28.5,29)$} & {$[29,29.5)$} & {$[29.5,30)$} & {$[30,30.5)$} & {$[30.5,31)$} & {$[31,31.5]$} \\
\hline$m_{2}{ }^{\prime}$ & 0.0214 & 0.1359 & 0.3413 & 0.3413 & 0.1359 & 0.0214 \\
\hline
\end{tabular}

Table 2: $m_{2}\left(I_{2, k}\right)$ BPA, $k=1, \ldots, 6$

\begin{tabular}{ccccc}
\hline$j, k$ & $A_{i}=I_{1, j} \times I_{2, k}$ & $m^{\prime}\left(I_{1, j} \times I_{2, k}\right)$ & $R_{i}=f\left(I_{1, j} \times I_{2, k}\right)$ & $\rho\left(f\left(I_{1, j} \times I_{2, k}\right)\right)$ \\
\hline 1,1 & $A_{1}=[45,46) \times[28.5,29)$ & 0.0001 & $(4357.5,4444.2]$ & 0.0001 \\
1,2 & $A_{2}=[45,46) \times[29,29.5)$ & 0.0009 & $(4320.5,4405.7]$ & 0.0009 \\
1,3, & $A_{3}=[45,46) \times[29.5,30)$ & 0.0023 & $(4284.3,4368.2]$ & 0.0023 \\
1,4 & $A_{4}=[45,46) \times[30,30.5)$ & 0.0023 & $(4249.0,4331.6]$ & 0.0023 \\
1,5 & $A_{5}=[45,46) \times[30.5,31)$ & 0.0009 & $(4214.6,4296.0]$ & 0.0009 \\
1,6 & $A_{6}=[45,46) \times[31,31.5]$ & 0.0001 & $(4181.1,4261.2]$ & 0.0001 \\
\hline 2,1 & $A_{7}=[46,47) \times[28.5,29)$ & 0.0006 & $(4310.9,4395.6]$ & 0.0006 \\
2,2 & $A_{8}=[46,47) \times[29,29.5)$ & 0.0038 & $(4274.3,4357.5]$ & 0.0038 \\
2,3, & $A_{9}=[46,47) \times[29.5,30)$ & 0.0095 & $(4238.5,4320.5]$ & 0.0095 \\
2,4 & $A_{10}=[46,47) \times[30,30.5)$ & 0.0095 & $(4203.6,4284.3]$ & 0.0095 \\
2,5 & $A_{11}=[46,47) \times[30.5,31)$ & 0.0038 & $(4169.6,4249.0]$ & 0.0038 \\
2,6 & $A_{12}=[46,47) \times[31,31.5]$ & 0.0006 & $(4136.3,4214.6]$ & 0.0006 \\
\hline$\vdots$ & $\vdots$ & $\vdots$ & & $\vdots$ \\
\hline 10,1 & $A_{55}=[54,55] \times[28.5,29)$ & 0.0001 & $(3985.1,4057.0]$ & 0.0001 \\
10,2 & $A_{56}=[54,55] \times[29,29.5)$ & 0.0009 & $(3951.2,4021.8]$ & 0.0009 \\
10,3 & $A_{57}=[54,55] \times[29.5,30)$ & 0.0023 & $(3918.1,3987.6]$ & 0.0023 \\
10,4, & $A_{58}=[54,55] \times[30,30.5)$ & 0.0023 & $(3885.9,3954.2]$ & 0.0023 \\
10,5 & $A_{59}=[54,55] \times[30.5,31)$ & 0.0009 & $(3854.4,3921.7]$ & 0.0009 \\
10,6 & $A_{60}=[54,55] \times[31,31.5]$ & 0.0001 & {$[3823.7,3889.9]$} & 0.0001 \\
\hline
\end{tabular}

Table 3 : Focal element and BPAs of the random relation $(\mathcal{F}, m)$ and its image $\left(\mathcal{R}^{\prime}, \rho^{\prime}\right) k=1, \ldots, 6$.

$$
\begin{aligned}
& P_{*}^{\zeta}(D)=F_{*}^{\zeta}(4210)-F_{\zeta}^{*}(4010)=0.5571 \\
& P_{\zeta}^{*}(D)=F_{\zeta}^{*}(4210)-F_{*}^{\zeta}(4010)=0.9233
\end{aligned}
$$

There is an inclusion relationship

$$
P_{*}^{\zeta}(D)=0.5571 \leq P_{\zeta}(D)=0.8057 \leq P_{\zeta}^{*}(D)=0.9233(5.14)
$$

Because $P_{\zeta}(D) \leq P_{\zeta}^{*}(D)<0.95$, system performance is unreliable. It is evident that the proposed method get same evaluation result with the one of the Monte Carlo method, but reduces the number of calculations required drastically.

In order to improve precision, let us divide $V=I_{1} \times I_{2}$ into more subintervals, i.e. $I_{1}$ is divided into $d_{1}=50$ subintervals, $I_{2}$ is divided into $d_{2}=44$ subintervals. $(50+1)$ $\times(44+1)=2295$ evaluations of $f$ are needed. The corresponding $F(y), F_{u p}(y)$ and $F_{\text {low }}(y)$ are showed in Fig 3. We can obtain again

$$
\begin{aligned}
& (1) F_{\zeta}^{*}(4010)=0.1007, F_{*}^{\zeta}(4010)=0.0737 \\
& \varepsilon_{-} r a n(4010)=0.0270 \\
& (2) F_{*}^{\zeta}(4210)=0.8780, F_{\zeta}^{*}(4210)=0.9079 \\
& \varepsilon_{-} r \text { ran }(4210)=0.0299
\end{aligned}
$$

(3) $P_{*}^{\zeta}(D)=0.7773, \quad P_{\zeta}^{*}(D)=0.8342$

Based on which, there is the inclusion relationship

$$
\begin{aligned}
& P_{*}^{\zeta}(D)=0.7773 \leq P_{\zeta}(D) \\
& =0.8057 \leq P_{\zeta}^{*}(D)=0.8342
\end{aligned}
$$

Because what we are interested in is only the probability of failure, not the entire distribution of $f$, it would have been necessary to pay attention to the errors at points $\zeta=4010 \mathrm{k} \mathrm{Hz}$ and $\zeta=4210 \mathrm{kHz}$ with respect to $F_{*}^{\zeta}$ (4010), $F_{\zeta}^{*}(4010), F_{*}^{\zeta}(4210)$ and $F_{\zeta}^{*}(4210)$. So let us chose the larger one in $\varepsilon_{-} \operatorname{ran}(4010)$ and $\varepsilon \_r a n(4210)$, i.e. $\varepsilon \_r a n(4210)=0.0299 \approx 0.03$ as our error. In order to obtain the same error with $99.99 \%$ confidence by means of Monte Carlo simulation, $N=89591318 \square 2295$ samplings would be required. Hence, if we set the error in advance and the inclusion $P_{*}^{\zeta}(D) \leq \Upsilon \leq P_{\zeta}^{*}(D)$ exists all the same, then we can conclude that system performance is in a limit state condition. 


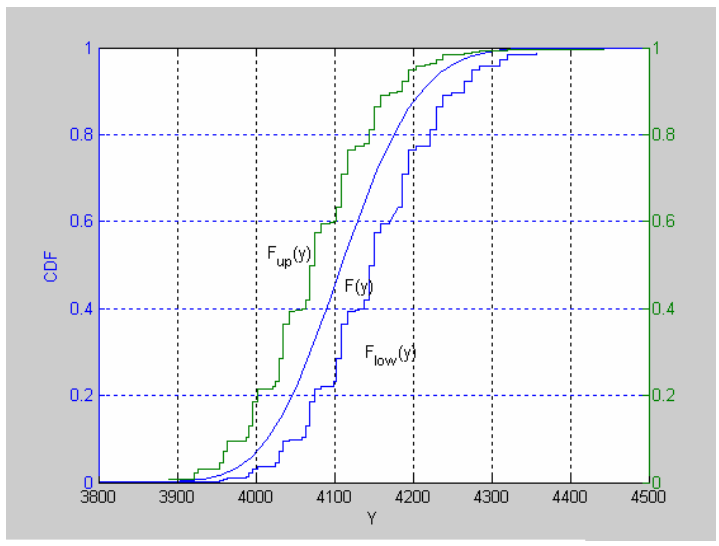

Figure 2: CDF and the upper and lower cumulative distribution of $f\left(d_{1}=10, d_{2}=6\right)$

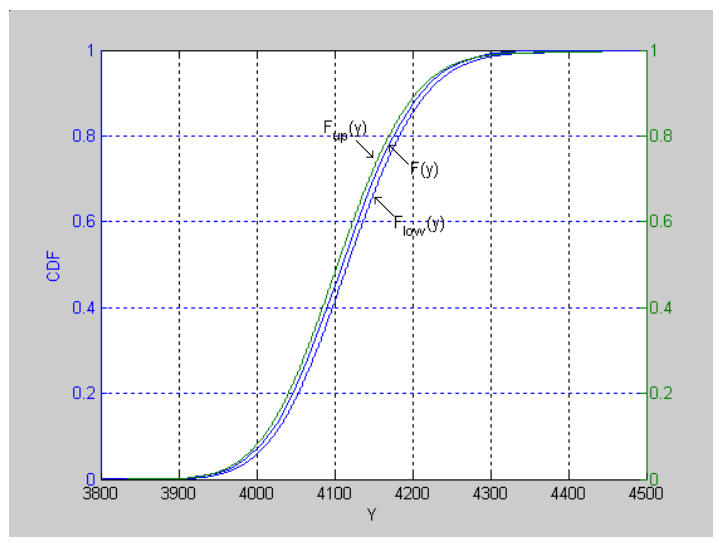

Figure3:CDF and the upper and lower cumulative distribution of $f\left(d_{1}=50, d_{2}=44\right)$

\section{Conclusions}

In this paper, random set theory is used to describe these parameter variables and obtain a simple and flexible method for evaluating reliability of circuit performance, which is alternative to Monte-Carlo analysis, but reduces the number of calculations required drastically. Here, we only consider the case that parameters is indpendent each other, and further more, when some parameters are correlative, how to generate upper and lower cumulative distribution should be studied based on random set theory.

\section{Acknowledgement}

This work is partially supported by the NSFC (Grant No.60434020, 60572051), Science and Technology Key Item of Ministry of Education of the PRC (No.205092), and the ZJNSF(Grant No..R106745).
[1] G Xiao, T.T Li.Monte Carlo methods for reliability analysis of system. Science press(China),2003.

[2] S.K Zeng, T.D. Zhao, J.G. Zhang,, Design and analysis of system reliability. Press of Beihang university,2001.

[3] X.T. Ling, Analysis and design of circuit parameters. Press of Fudan university,1991.

[4] X.Q. Li, D.Y. Gao,C.H. Liu,Y.H. Li, A high efficient exchange method in circuit analysis with Monte Carlo sampling. Journal of Xian university, 26(1):112-114, 1999.

[5] D.L. Peng, C.L. Wen, X.B.Xu,A.K. Xue, Random Set and Its Applications in Information Fusion.Journal of Electronics \& Information Technology, 28(11):2199-2204,2006.

[6] I. R. Goodman, R. P. S. Mahler, and H. T. Nguyen. Mathematics of Data Fusion. Kluwer Academic Publishers, AA Dordrecht, the Netherlands, ISBN 0-7923-4674-2.1997.

[7] D. Dubois,H. Prade. Random sets and fuzzy interval analysis. Fuzzy Sets and Systems,42:87101, 1991

[8] G. Shafer, A Mathematical Theory of Evidence. Princeton University Press, 1976.

[9] J.Khlas, Modeling uncertainty with belief functions in numerical models, Rep. No. 141, Instit. for Automation and Operations Research, Univ. of Fribourg, Switzerland, 1987.

[10] H. Ratschek, J. Rokne. New computer methods for global optimization. Chirchester: Ellis Horwood, 1988.

[11] RE Moore. Interval analysis. Englewood Cliffs, NJ: Prentice Hall,1966.

[12] W.M. Dong, H.C. Shah. Vertex method for computing functions of fuzzy variables. Fuzzy Sets and Systems,24:65-78,1987.

[13] F. Tonon, A. Bemardini. A random set approach to the optimization of uncertain structures. Computers and Structures,;68(6):583-600, 1998

[14] D. Dubois and H. Prade, A set-theoretic view of belief functions. Logical operations and approximations by fuzzy sets. Int.J.General Systems, 12:193-226,1986.

[15] A. Kolmogorov. Confidence limits for an unknown distribution function.Annals of Mathematical Statistics,12(4):461-3, 1941.

\section{References}

